\title{
Development of an immunochromatographic strip for the serodiagnosis of Theileria infection in sheep
}

Yizhu Lu', Guiquan Guan', Tao Jiang ${ }^{1}$, Youquan Li ${ }^{1}$, Jifei Yang ${ }^{1}$, Guangyuan Liu' ${ }^{1}$ Jianxun Luo ${ }^{1}$, Hong Yin ${ }^{1,2}$ and Zhijie Liu ${ }^{1 *}$

\begin{abstract}
Background: Theileria uilenbergi and T. luwenshuni are tick-borne protozoan parasites, transmitted by Haemaphysalis qinghaiensis and $\mathrm{H}$. longicornis, respectively. They are the main causative agents of theileriosis in small ruminants in China. The disease has resulted in severe economic losses and hindered the development of sheep and goat husbandry industry in the endemic regions.

Methods: In this study, a colloidal gold-based immunochromatographic strip (ICS) was developed for the detection of T. uilenbergi and/or T. luwenshuni infections. A recombinant T. uilenbergi immunodominant protein (rTulP) was used as antigen for the ICS. The nitrocellulose membrane was incubated with rTulP on the test (T) line and anti-rTulP antiserum on the control (C) line, respectively. The rTulP conjugated to colloidal gold particles was used as the detection system for visualization of the lines. Then the sample pad, conjugate pad, nitrocellulose membrane and absorbent pad were assembled onto a backing plate in the appropriate order.

Results: The ICS was able to detect antibodies in the sera of animals experimentally infected with T. uilenbergi from 14 to 85 days. It also reacted with the serum from T. luwenshuni infected sheep. However, there was no cross-reactivity with sera from animals infected with Babesia motasi and Anaplasma ovis. Comparison of the ICS with the rTuIP antigen based indirect enzyme-linked immunosorbent assays (ELISA) using test field samples showed good correlations with $93.1 \%(81 / 87)$ sensitivity and $100 \%$ (40/40) specificity, respectively, with an almost perfect agreement (Kappa = 0.895, $P<0.01)$.

Conclusion: An immunochromatographic strip test based on a recombinant T. uilenbergi immunodominant protein (rTulP) was developed. This is a rapid test (approximately $15 \mathrm{~min}$ to completion) for the detection of $T$. uilenbergi and/or T. luwenshuni infection that is easy to perform and; delivers results that are visible to the naked eye.
\end{abstract}

Keywords: Theileria uilenbergi, Theileria luwenshuni, Colloidal gold, Immunochromatographic strip test

\section{Background}

Theileria uilenbergi and T. luwenshuni are tick-borne protozoan parasites. They are causative pathogens of theileriosis of small ruminants in China [1]. The known transmission vectors for both parasites are $H$. qinghaiensis and $H$. longicornis [2-4]. Occurrence of theileriosis

\footnotetext{
* Correspondence: liuzhijie@caas.cn

'State Key Laboratory of Veterinary Etiological Biology, Key Laboratory of Veterinary Parasitology of Gansu Province, Lanzhou Veterinary Research Institute, Chinese Academy of Agricultural Sciences, Xujiaping 1, Lanzhou, Gansu 730046, P. R. China

Full list of author information is available at the end of the article
}

has been reported in many regions in China, including Gansu, Qinghai, Sichuan, Liaoning, Shanxi, Inner Mongolia, Ningxia, Xinjiang and Hubei provinces [5-9]. The disease mainly causes fever, anemia, icterus and can be fatal in goats and sheep. As such, it has restricted the development and productivity of the small ruminant livestock industry in the endemic regions [7].

The most practical and commonly used method for diagnosis of theileriosis by the veterinarian is examination of piroplasm of T. uilenbergi and/or T. luwenshuni in Giemsa-stained blood smears using a light microscope 
[10]. This method is reliable for the detection of infection in acute cases, but it is limited for the diagnosis of chronic cases because of the low number of parasitemia in ruminants [11]. Additionally, it is impossible to distinguish T. uilenbergi from T. luwenshuni due to their similar morphological shape. In recent years, nuclear acid based diagnostic methods have been developed to efficiently detect and/or differentiate the 2 parasites, such as polymerase chain reaction (PCR) [12-15], reverse line blot (RLB) hybridization assays [16, 17], multiplex PCR (mPCR) [18] and loop-mediated isothermal amplification (LAMP) [19]. Although these provide reliable and unambiguous pathogen detection methods for the diagnosis of the infection, they are impractical for field diagnosis since equipped laboratories are required. Regarding serological tests, several ELISAs have been established. The ELISA based on crude antigen (merozoite lysate) can detect antibodies against both $T$. uilenbergi and T. luwenshuni [20]; however preparation and standardization of the crude antigen is difficult. Alternatively, an ELISA based on the recombinant protein rTuIP has been developed and validated, which is suitable for epidemiological studies and large-scale studies [21, 22]. However, the rTuIP based ELISA is still limited as it is a laboratory test that requires professional personnel, special laboratory materials and equipment. Hence, a convenient and rapid test, such as an immunochromatographic strip is needed for the use in both clinical and field applications for the diagnosis of ovine theileriosis in China.

The colloidal gold-based immunochromatographic strip is easy to perform in the field and does not require expensive instruments. It has been widely used for the detection of infection with pathogens such as T. annulata, canine parvovirus, Leptospira, Trichinella in the veterinary field [23-26]. The aim of the present study was to develop a simple, portable and rapid immunochromatographic strip for the detection of T. uilenbergi and/or T. luwenshuni infections in sheep and goats.

\section{Methods}

\section{Source of serum samples}

Serum samples were prepared as described previously [21]. Theileria-free sheep were examined for merozoites in Giemsa-stained blood smears under the microscope and by PCR detection. Negative serum samples were collected before experimental infection. Sheep (No. 2203, 1236, 1219) were inoculated using blood-infected with T. uilenbergi; sheep (No. 1250, 1240, 1229) were attached with ticks collected in a Theileria-endemic region (Lintan). Serum samples were taken at 14, 19, 52 and 85 days, post infection.

One hundred and twenty seven serum samples were collected at random from Lintan County, in
Gansu Province, which had been tested previously by using both the merozoite antigen ELISA and rTuIP ELISA [20, 21]. Positive sera of T. luwenshuni, $B$. motasi and $A$. ovis were prepared from T. luwenshuni, $B$. motasi and $A$. ovis-infected sheep, respectively, as described previously [21].

\section{Ethical approval}

This study was approved by the Animal Ethics Committee of the Lanzhou Veterinary Research Institute. Care and maintenance of animals was in accordance with institutional guidelines of the Lanzhou Veterinary Research Institute, Chinese Academy of Agricultural Sciences.

\section{Preparation of antigen and polyclonal antibodies}

Recombinant TuIP was prepared as described by Liu et al. [21]. Briefly, N-terminal region of the TuIP gene was cloned into the $\mathrm{pQE} 31$ expression vector, generating in a recombinant protein of 382 amino acids with a predicted molecular mass of $41.7 \mathrm{kDa}$. The protein product was termed rTuIP as previous reported [21]. Subsequently, the rTuIP protein was purified according to the QiaExpressionist protocols (Qiagen, Hilden, Germany). Rabbit anti-rTuIP polyclonal antibody was prepared as reported in a previous study [21].

\section{Preparation of the ICS}

Colloidal gold was prepared according to the published method [27]. Briefly, $2 \mathrm{~mL}$ of $1 \%$ trisodium citrate (w/v) was added quickly to $100 \mathrm{~mL}$ of $0.01 \% \mathrm{HAuCl} 4$ solution (w/v) heated to $90{ }^{\circ} \mathrm{C}$ and boiled for 15 min under constant stirring. As the solution cooled to room temperature (RT), the $\mathrm{pH}$ was adjusted to 9.0 using $0.01 \mathrm{M}$ potassium carbonate and sodium azide added to a final concentration of $0.02 \%(\mathrm{w} / \mathrm{v})$. Two hundred microliter of rTuIP $(2 \mathrm{mg} / \mathrm{ml})$ was added to $20 \mathrm{ml} \mathrm{col-}$ loidal gold solution and stirred $15 \mathrm{~min}$ followed by $4 \mathrm{ml}$ $5 \%$ BSA to block non-specific binding sites. The resulting colloidal gold-rTuIP was centrifugated at 10,000 rpm $40 \mathrm{~min}$ and the resuspended pellet in $1.2 \mathrm{ml}$ of resuspension buffer was sprayed onto glass fiber pads and, then dried at $37{ }^{\circ} \mathrm{C} 30 \mathrm{~min}$. Nitrocellulose membrane was incubated with rTuIP at the $\mathrm{T}$ line and an anti-rTuIP antibody at the $\mathrm{C}$ line and then dried at $37{ }^{\circ} \mathrm{C}$ for $30 \mathrm{~min}$. The sample pad, conjugate pad, nitrocellulose membrane and absorbent pad were assembled on a backing plate in the appropriate order [26]. The finished plate was cut into $2.5 \mathrm{~mm} \times 80 \mathrm{~mm}$ strips using a cutting machine. In a test, the sample was added to the sample pad where the liquid migrated towards the conjugated pad. The antibody was captured by the colloidal gold-rTuIP on the conjugated pad and resulting complex migrated to the next section of the strip which was the reaction matrix. If the sample contained anti-Theileria antibodies, 
the complex would react with the immobilized rTuIP antigen on the "T" line and a positive signal would be indicated with a red band. If the sample did not contain anti-Theileria antibodies, the free conjugate would migrate along the membrane towards the " $\mathrm{C}$ " line where it would interact with immobilized anti-rTuIP antibodies. The rest of the solution is trapped in the absorbent pad. If both the " $\mathrm{T}$ " line and the " $\mathrm{C}$ " line formed color bands, the sample was considered positive. If only the " $\mathrm{C}$ " line but not the " $\mathrm{T}$ " line formed color band, the sample was considered to be negative. The absence of a " $\mathrm{C}$ " line would indicate test failure.

\section{Preliminary evaluation of the ICS}

To test specificity of the ICS, positive sera from $T$. luwenshuni, B. motasi and A. oivs and negative sera from healthy sheep were tested. The sensitivity of the ICS was evaluated with positive sera which was diluted at 1:50, $1: 100,1: 200,1: 250,1: 300$, and 1:500.

Field samples $(n=127)$ were tested with the ICS and compared to previously generated data using rTuIP based ELISA [21]. The correlation of the two methods was evaluated in terms of the sensitivity and specificity using the following formulas: sensitivity $(\mathrm{Se}=$ (No. of samples positive in both tests/total number of positive samples in the reference test $) \times 100)$, specificity $(S p=($ No. of samples negative in both tests/total number of negative samples in the reference test) $\times 100$ ). The Kappa test was used to calculate the degree of agreement between the ICS and rTuIP ELISA using SPSS 18.0.0 software for Windows (SPSS Inc., Chicago, IL, USA) [28].

\section{Results}

\section{Establishment of the ICS}

A range of concentration of colloidal gold-rTuIP on the conjugated pad, rTuIP on the "T" line and rabbit antirTuIP polyclonal antibody on the "C" line were used to optimize the ICS system. The optimal concentration of the colloidal gold-rTuIP on glass fiber pads and the
rTuIP on the "T" line were $0.02 \mathrm{mg} / \mathrm{ml}$ and $0.5 \mathrm{mg} / \mathrm{ml}$, respectively. A dilution of 1:15 was found to be optimal for the rabbit anti-rTuIP polyclonal antibody on the "C" line for the subsequent experiment. For testing serum samples, 50 to $100 \mu \mathrm{l}$ samples were loaded onto the sample pad, and the result was visually detected to the naked eye within $15 \mathrm{~min}$.

\section{Evaluation of the ICS}

Analysis of serum samples collected at days, 0, 14, 19, 52 and 85 from the experimentally infected animals was done using the ICS. The results demonstrated that the ICS was able to detect antibodies from days 14 to 85 (Table 1), indicating that the test is suitable for the detection of T. uilenbergi infection.

When sera from sheep experimentally infected with $T$. luwenshuni, B.motasi and A. ovis were tested with the ICS, a positive cross-reaction was observed from serum from the closely related pathogen, $T$. luwenshuni. No cross-reaction was observed from negative serum or positive sera of sheep experimentally infected with $B$. motasi and A. ovis (Fig. 1a). To assess analytical sensitivity, positive serum samples diluted at 1:50, 1:100, 1:200, 1:250, 1:300, and 1:500 were tested with the ICS. The red line could be observed clearly at both the $\mathrm{T}$ and $\mathrm{C}$ lines when the dilution of the serum samples was no less than 1:200. A weak red line at the $\mathrm{T}$ line could still be detected at 1:250 dilution. This result suggested that the ICS could detect antibodies in serum samples at very low concentrations (Fig. 1b).

In a preliminary run, the ICS was evaluated for its reliability by testing anti-TuIP-specific antibodies against 127 field samples under optimized conditions, and comparing the results obtained with serum samples using TuIP-ELISA [21]. When compared to the TuIP-ELISA as reference test, the correlation in terms of the sensitivity and specificity was $93.10 \%(81 / 87)$ and $100 \%$ (40/40), respectively, with an almost perfect agreement (Kappa 0.895, $P<0.01$ ) (Table 2).

Table 1 Detection of anti-TulP specific antibody in sera from sheep experimentally infected with T. uilenbergi taken at daily intervals post-infection using the immunochromatographic strip

\begin{tabular}{|c|c|c|c|c|c|c|}
\hline & \multicolumn{6}{|c|}{ Experimental infected sheep } \\
\hline & \multicolumn{3}{|c|}{ Infectious blood inoculation } & \multicolumn{3}{|c|}{ Infected $H$. qinghaiensis infestation } \\
\hline & 2203 & 1236 & 1219 & 1250 & 1240 & 1229 \\
\hline 0 days p.i. & $+/-$ & - & - & - & - & - \\
\hline 14 days p.i. & + & + & + & + & + & + \\
\hline 19 days p.i. & + & + & + & + & + & + \\
\hline 52 days p.i. & + & + & + & + & + & + \\
\hline 85 days p.i. & + & + & + & + & + & + \\
\hline
\end{tabular}

"+" indicates a positive result, "-" a negative result, "+/-" a suspected result, p.i. post infection, Sheep Nos. 1250, 1240 and 1229 were infected by feeding 200 adult Heamaphysalis qinghaiensis ticks collected from Lintan, China, Sheep Nos. 2203, 123681 and 1219 were infected by blood inoculation with T. uilenbergi Lintan isolate 


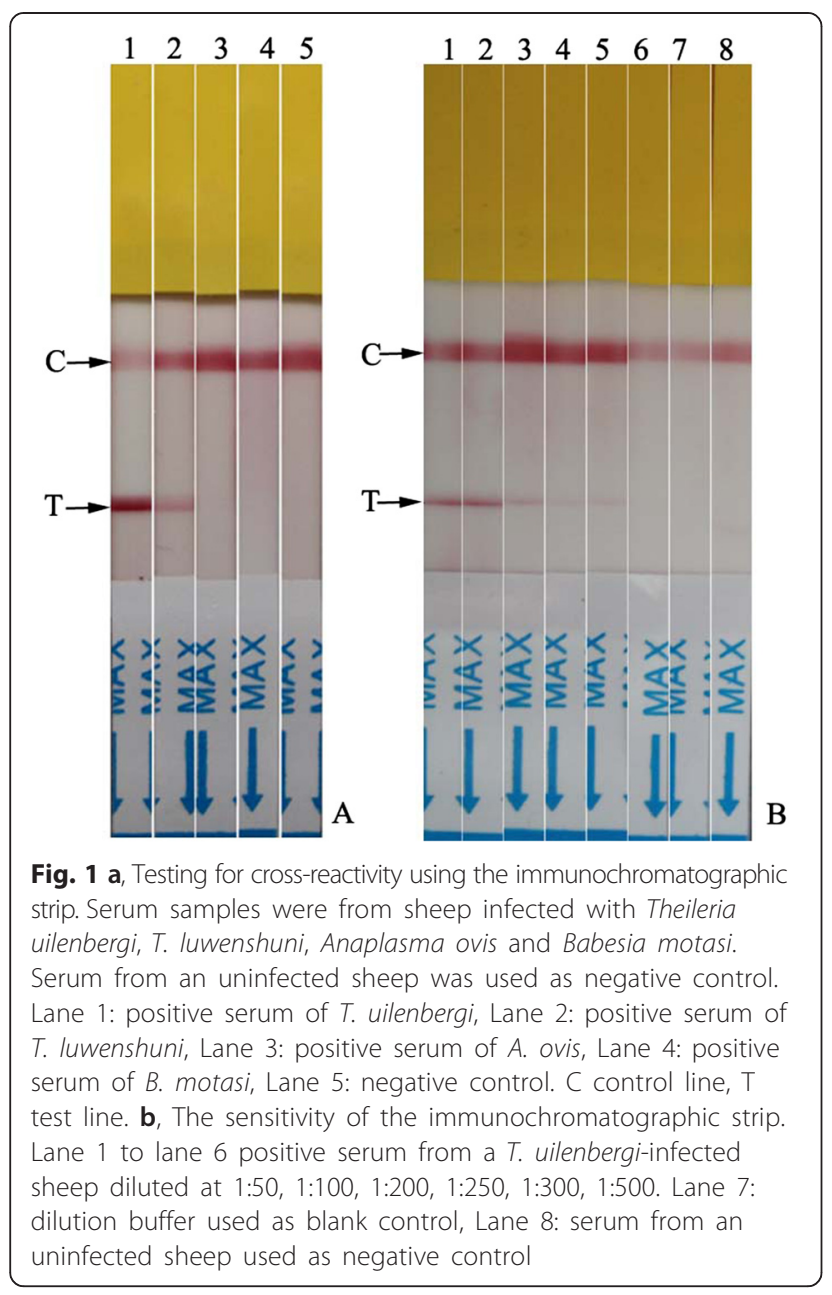

\section{Discussion}

The ICS is a portable and rapid method for the detection of antibodies in serum and has been widely applied for the diagnosis of protozoa parasite infections, such as Babesia [29-31], Toxoplasma [32, 33]. The merits of ICS include; increased speed, it is simple to use, and it is easy-to-conduct on site with no requirement of laboratory instruments. Moreover, the result is visible and easily detected by the naked eye. Despite these advantages, the ICS has not yet been developed for the detection of T. uilenbergi and T. luwenshuni infection.

TuIP is an antigen of T. uilenbergi, and the recombinant TuIP has been successfully used for the development of an indirect ELISA for the detection of T. uilenbergi and T. luwenshuni infection [21, 22]. The rTuIP was therefore chosen as an antigen to develop the ICS in this study. Besides the antigen, several critical aspects in the establishment of an ICS had to be considered, such as the quality and concentration of antigen, antibody, and/or colloidal gold particles, and the buffer system $[23,34]$. To optimize the ICS, different concentration of the colloidal gold-rTuIP on the conjugated pad,
Table 2 Comparison of the immunochomatographic strip with the rTUIP-ELISA as the reference test for detection of antibodies in 127 field samples from sheep

\begin{tabular}{lllll}
\hline & & \multicolumn{2}{l}{ rTulP-ELISA } & \\
\cline { 3 - 5 } & & Positive & Negative & Total \\
\hline ICS & Positive & 81 & 0 & 81 \\
& Negative & 6 & 40 & 46 \\
Total & 87 & 40 & 127 \\
\hline
\end{tabular}

Sensitivity of the ICS $=93.1 \%$; specificity of the ICS $=100 \%$; measure of agreement of the two tests, Kappa $=0.895, P<0.01$

rTuIP antigen on the " $\mathrm{T}$ " line and rabbit anti-rTuIP polyclonal antibody on the "C" line were tested.

When testing for potential cross-reactivity of the ICS using T. luwenshuni, B. motasi and A. ovis, the results showed that the ICS could also detect $T$. luwenshuni infection, but not Babesia and Anaplasma infections. This is accordance with the results obtained from the rTuIP based ELISA in our previous studies [21, 22]. It is known that T. uilenbergi and T. luwenshuni are very closely related pathogens in terms of morphology, transmission vectors, hosts, and distribution in the endemic regions as well as phylogenetic relationship [35, 36]. Therefore it was assumed that TuIP of T. uilenbergi might share similar epitopes in T. luwenshuni [22].

Testing of the serum samples from experimentally infected sheep by time course at days 14,19,52,85 gave positive results that correlated to the rTuIP based ELISA [21]. These results indicate that the ICS is suitable for the detection of the infection caused by the Theileria parasites both during the early stage and the chronic phase. Further evaluation of ICS was done by testing 127 field serum samples and comparing the results with data obtained by investigation of serum samples using the rTuIP based ELISA. The comparison showed that the ICS had an almost perfect agreement to the rTuIP based ELISA (Kappa $=0.895, P<0.01$ ) in terms of the sensitivity of $93.1 \%$ and the specificity of $100 \%$, indicating that the ICS would be reliable for use in the field.

\section{Conclusion}

An immunochromatographic strip based on a recombinant $T$. uilenbergi immunodominant protein (rTuIP) was developed for the detection of T. uilenbergi and/or $T$. luwenshuni infection in the field. The sensitivity and specificity were in good agreement with the rTuIP-based indirect ELISA [21]. This method is not able to differentiate $T$. uilenbergi infection from $T$. luwenshuni infection, however it is easily applicable by veterinarians for the onsite diagnosis of ovine theileriosis.

\section{Competing interests}

The authors declare that they have no competing interest. 


\section{Authors' contributions}

$Z L, J L$ and $H Y$ participated in the design of study and coordination. YLu and YLi contributed to recombinant TulP preparation, establishment of immunochromatographic strip. GG and JF supervised and participated in evaluation of the strip. TJ and GL carried out the preparation of gold particles. YLu and ZL did statistical analysis. YLu, GG and ZL drafted the manuscript. All authors read and approved the final manuscript.

\section{Acknowledgments}

This study was financially supported by the NSFC (No. 31372432, No. 31001061, No. 31272556), ASTIP, Creative Research Groups of Gansu Province (No. 1210RJIA006), NBCIS CARS-38, Special Fund for Agro-scientific Research in the Public Research (No. 201303035, No. 201303037), MOA, Specific Fund for Sino-Europe Cooperation, MOST, China. The research was also facilitated by PIROVAC (KBBE-3-245145) of European Commission.

\section{Author details}

'State Key Laboratory of Veterinary Etiological Biology, Key Laboratory of Veterinary Parasitology of Gansu Province, Lanzhou Veterinary Research Institute, Chinese Academy of Agricultural Sciences, Xujiaping 1, Lanzhou, Gansu 730046, P. R. China. ${ }^{2} J i a n g s u$ Co-innovation Center for Prevention and Control of Important Animal Infectious Diseases, Yangzhou, China.

\section{Received: 30 June 2015 Accepted: 30 November 2015} Published online: 02 December 2015

\section{References}

1. Yin H, Schnittger L, Luo J, Seitzer U, Ahmed JS. Ovine theileriosis in China: a new look at an old story. Parasitol Res. 2007;101 Suppl 2:S191-5.

2. Yin H, Luo J, Guan G, Gao Y, Lu B, Zhang Q, et al. Transmission of an unidentified Theileria species to small ruminants by Haemaphysalis qinghaiensis ticks collected in the field. Parasitol Res. 2002;88:S25-7.

3. Li Y, Luo J, Liu Z, Guan G, Gao J, Ma M, et al. Experimental transmission of Theileria sp. (China 1) infective for small ruminants by Haemaphysalis longicornis and Haemaphysalis qinghaiensis. Parasitol Res. 2007:101:533-8.

4. Li Y, Luo J, Guan G, Ma M, Liu A, Liu J, et al. Experimental transmission of Theileria uilenbergi infective for small ruminants by Haemaphysalis longicornis and Haemaphysalis qinghaiensis. Parasitol Res. 2009;104:1227-31.

5. Yang F, Feng Z, Yu G, Liu J, Wei Z, He X. A report on ovine theileriosis in Ganning animal farm station in Ganzi district. Chin J Vet Med. 1985;2:33-7.

6. Wang F, Li O, Li W, Wang W, Hua D, Wang L, et al. Investigation on Theileria hirci in Huangyuan, Menyuan and Jianzha counties. Newsl Vet Med Anim Husb(Qinghai). 1980;3:29-32.

7. Luo J, Yin H. Theileriosis of sheep and goats in China. Trop Anim Health Prod. 1997:29:S8-10.

8. Ge Y, Pan W, Yin H. Prevalence of Theileria infections in goats and sheep in southeastern China. Vet Parasitol. 2011;186:466-9.

9. Li Y, Peng Y, Liu J, Guan G, Yang J, Chen Z, et al. Epidemiological survey and identification of Theileria parasite infection for small ruminants in some parts of China. Sci Agric Sin. 2012;45:3422-9.

10. Criado-Fornelio A. M A. Gónzalez-del-R’ıo, A Buling-Saraña, J.C. Barba- Carretero. The "expanding universe" of piroplasms. Vet Parasitol. 2007;119:337-45.

11. Zweygarth $\mathrm{E}$, Just MC, De Waal DT. In vitro cultivation of Babesia equi: detection of carrier animals and isolation of parasites. Onderstepoort J Vet Res. 1997;64:51-6.

12. Allsopp BA, Baylis HA, Allsopp MT, Cavaller-Smith T, Bishop RP, Carrington DM, et al. Discrimination between six species of Theileria using oligonucleotide probes which detect small subunit ribosomal RNA sequences. Parasitology. 1993;107:157-65.

13. Schnittger L, Yin H, Jianxun L, Ludwig W, Shayan P, Rahbari S, et al. Ribosomal small-subunit RNA gene-sequence analysis of Theileria lestoquardi and a Theileria species highly pathogenic for small ruminants in China. Parasitol Res. 2000:86:352-8.

14. Sun C, Liu Z, Gao J, Guan G, Ma M, Luo J, et al. Investigations into the natural infection rate of Haemaphysalis qinghaiensis with Piroplasma using a nested PCR. Exp Appl Acarol. 2008;44:107-14.
15. Yin H, Liu Z, Guan G, Liu A, Ma M, Ren Q, et al. Detection and differentiation of Theileria luwenshuni and T. uilenbergi infection in small ruminants by PCR. Transbound Emerg Dis. 2008;55:233-7.

16. Niu Q, Luo J, Guan G, Ma M, Liu Z, Liu A, et al. Detection and differentiation of ovine Theileria and Babesia by reverse line blotting in China. Parasitol Res. 2009;104:1417-23.

17. Schnittger L, Yin H, Qi B, Gubbels MJ, Beyer D, Niemann S, et al. Simultaneous detection and differentiation of Theileria and Babesia parasites infecting small ruminants by reverse line blotting. Parasitol Res. 2004;92:189-96

18. Zhang X, Liu Z, Yang J, Chen Z, Guan G, Ren Q, et al. Multiplex PCR for diagnosis of Theileria uilenbergi, Theileria luwenshuni, and Theileria ovis in small ruminants. Parasitol Res. 2014;113:527-31.

19. Liu Z, Hou J, Bakheit MA, Salih DA, Luo J, Yin H, et al. Development of loop-mediated isothermal amplification (LAMP) assay for rapid diagnosis of ovine theileriosis in China. Parasitol Res. 2008;103:1407-12.

20. Gao YL, Yin H, Luo JX, Ouyang WQ, Bao HM, Guan GQ, et al. Development of an enzyme-linked immunosorbent assay for the diagnosis of Theileria sp infection in sheep. Parasitol Res. 2002;88:S8-10.

21. Liu Z, Wang Z, Yin H, Luo J, Zhang B, Kullmann B, et al. Identification of Theileria uilenbergi immunodominant protein for development of an indirect ELISA for diagnosis of ovine theileriosis. Int J Parasitol. 2010;40:591-8

22. Liu Z, Li Y, Salih DE, Luo J, Ahmed JS, Seitzer U, et al. Validation of a recombinant protein indirect ELISA for the detection of specific antibodies against Theileria uilenbergi and Theileria luwenshuni in small ruminants. Vet Parasitol. 2014:204:139-45.

23. Abdo J, Kristersson T, Seitzer U, Renneker S, Merza M, Ahmed J. Development and laboratory evaluation of a lateral flow device (LFD) for the serodiagnosis of Theileria annulata infection. Parasitol Res. 2010;107(5):1241-8.

24. Oh JS, Ha GW, Cho YS, Kim MJ, An DJ, Hwang KK, et al. One-step immunochromatography assay kit for detecting antibodies to canine parvovirus. Clin Vaccine Immunol. 2006;13(4):520-4.

25. Chirathaworn C, Janwitthayanan W, Sereemaspun A, Lertpocasombat K, Rungpanich $U$, Ekpo P, et al. Development of an immunochromatographic test with anti-LipL32-coupled gold nanoparticles for Leptospira detection New Microbiol. 2014;37(2):201-7.

26. Zhang GP, Guo JQ, Wang XN, Yang JX, Yang YY, Li QM, et al. Development and evaluation of an immunochromatographic strip for trichinellosis detection. Vet Parasitol. 2006;137(3-4):286-93.

27. Yang S, Yang J, Zhang G, Wang X, Qiao S, Zhao D, et al. Development of an immunochromatographic strip for the detection of antibodies against foot-and-mouth disease virus serotype. J Virol Methods. 2010;165(2):139-44.

28. Thomas EE, Puterman ML, Kawano E, Curran M. Evaluation of seven immunoassays for detection of rotavirus in pediatric stool samples. J Clin Microbiol. 1988:26:1189-93.

29. Huang X, Xuan X, Xu L, Zhang S, Yokoyama N, Suzuki N, et al. Development of an immunochromatographic test with recombinant EMA-2 for the rapid detection of antibodies against Babesia equi in horses. J Clin Microbiol. 2004;42:359-61.

30. Kim CM, Alhassan A, Verdida RA, Yokoyama N, Xuan X, Fujisaki K, et al. Development of two immunochromatographic tests for the serodiagnosis of bovine babesiosis. Vet Parasitol. 2007:148:137-43.

31. Kim CM, Blanco LB, Alhassan A, Iseki H, Yokoyama N, Xuan X, et al. Development of a rapid immunochromatographic test for simultaneous serodiagnosis of bovine babesioses caused by Babesia bovis and Babesia bigemina. Am J Trop Med Hyg. 2008;78:117-21.

32. Huang X, Xuan X, Hirata H, Yokoyama N, Xu L, Suzuki N, et al. Rapid immunochromatographic test using recombinant SAG2 for detection of antibodies against Toxoplasma gondii in cats. J Clin Microbiol. 2004:42:351-3.

33. Wang YH, Li XR, Wang GX, Yin H, Cai XP, Fu BQ, et al. Development of an immunochromatographic strip for the rapid detection of Toxoplasma gondii circulating antigens. Parasitol Int. 2011;60:105-7.

34. Zhang GP, Wang XN, Yang JF, Yang YY, Xing GX, Li QM, et al. Development of an immunochromatographic lateral flow test strip for detection of beta-adrenergic agonist clenbuterol residues. J Immunol Methods. 2006:312:27-33. 
35. Schnittger L, Yin H, Gubbels MJ, Beyer D, Niemann S, Jogejan F, et al. Phylogeny of sheep and goat Theileria and Babesia parasites. Parasitol Res. 2003;91(5):398-406.

36. Yin H, Luo J, Schnittger L, Lu B, Beyer D, Ma M, et al. Phylogeny of analysis of Theileria species transmitted by Haemaphysalis qinghaiensis. Parasitol Res. 2004;92:36-42.

Submit your next manuscript to BioMed Central and we will help you at every step:

- We accept pre-submission inquiries

- Our selector tool helps you to find the most relevant journal

- We provide round the clock customer support

- Convenient online submission

- Thorough peer review

- Inclusion in PubMed and all major indexing services

- Maximum visibility for your research 